\title{
On the metal abundances inside mixed-morphology supernova remnants: the case of IC 443 and $\mathrm{G} 166.0+4.3$
}

\author{
F. Bocchino ${ }^{1}$, M. Miceli $^{2}$, and E. Troja ${ }^{3,4}$ \\ 1 INAF - Osservatorio Astronomico di Palermo, Piazza del Parlamento 1, 90134 Palermo, Italy \\ e-mail: bocchino@astropa.inaf.it \\ 2 Consorzio COMETA, via S. Sofia 64, 95123 Catania, Italy \\ 3 Dipartimento di Scienze Fisiche ed Astronomiche, Sezione di Astronomia, Università di Palermo, Piazza del Parlamento 1, \\ 90134 Palermo, Italy \\ 4 INAF - Istituto di Astrofisica Spaziale e Fisica Cosmica, Sezione di Palermo, via Ugo la Malfa 153, 90146 Palermo, Italy \\ Received 4 August 2008 / Accepted 10 January 2009
}

ABSTRACT

\begin{abstract}
Context. Recent developments in the study of mixed morphology supernova remnants (MMSNRs) have revealed the presence of metal-rich X-ray emitting plasma inside a fraction of these remnants, a feature not properly addressed by traditional models for these objects.

Aims. Radial profiles of thermodynamical and chemical parameters are needed for a fruitful comparison of data and model of MMSNRs, but these are available only in a few cases.

Methods. We analyzed XMM-Newton data of two MMSNRs, namely IC 443 and G166.0+4.3, previously known to have solar metal abundances, and performed a spatially resolved spectral analysis of the X-ray emission.

Results. We detected enhanced abundances of $\mathrm{Ne}, \mathrm{Mg}$ and $\mathrm{Si}$ in the hard X-ray bright peak in the north of IC 443, and of sulphur in the outer regions of G166.0+4.3. The metal abundances are not distributed uniformly in both remnants. The evaporating clouds model and the radiative SNR model fail to reproduce consistently all the observational results.

Conclusions. We suggest that further deep X-ray observations of MMSNRs may reveal more metal-rich objects. More detailed models including ISM-ejecta mixing are needed to explain the nature of this growing subclass of MMSNRs.
\end{abstract}

Key words. ISM: supernova remnants - ISM: dust, extinction - X-rays: ISM - X-rays: individuals: IC 443 X-rays: individuals: G166.0+4.3 - ISM

\section{Introduction}

Mixed-morphology supernova remnants (MMSNRs) have been traditionally defined as remnants with a shell morphology in radio and a centrally peaked morphology in the X-ray band characterized by a thermal spectrum (Rho \& Petre 1998). The origin of this atypical morphology is controversial. Traditional models rely mostly on the effects of thermal conduction (e.g., Cox et al. 1999; Shelton et al. 1999; White \& Long 1991), but there are also models that invoke projection effects (Petruk 2001). Tilley et al. (2006) attempted to improve previous analytical models by using a set of hydrodynamical simulations designed to reproduce the morphology observed in MMSNRs.

In parallel to these theoretical efforts, there is growing observational interest about MMSNRs, which has led in a few cases to useful comparisons with models. Slane et al. (2002) favor the evaporating clouds White \& Long (1991) model for G290.1-0.8 (rather then the radiative model of Shelton et al. 1999), but problems remain in the comparison of the surface brightness profiles. Lazendic \& Slane (2006) also found a reasonable agreement between both models and X-ray observations of CTB1 and HB21, but they pointed out that the predicted central density was below the derived densities of the emiting plasma. In contrast, Rho \& Borkowski (2002) argued that the White \& Long (1991) evaporating cloud model cannot explain the strong radiative shock front in the MMSNR W28, and that the radiative model could not reproduce the strong thermal variation found in this remnant (see also Chevalier 1999, for a critical analysis of the evaporating cloud model of MMSNRs).

Several authors proposed that some of the X-ray emission of MMSNRs may be due to thermal plasma of high metal abundances, as in the case of stellar ejecta inside young historical SNRs (e.g., Shelton et al. 2004; Chen et al. 2008), or in other Galactic (e.g., Vela SNR, Miceli et al. 2008; Cygnus Loop, Katsuda et al. 2008; Puppis A, Hwang et al. 2008) and Magellanic Cloud SNRs (e.g., 0103-72.6, Park et al. 2003a; N49B, Park et al. 2003b). This important result was addressed and summarized by Lazendic \& Slane (2006), who compiled a sample of 26 MMSNRs, 10 of which appear to show enhanced metal abundances in their X-ray spectrum. We emphasize that the presence of enhanced metal abundances inside MMSNRs has not been addressed in detail in models of this sub-class of remnants. Published models with thermal conduction do not yet take account of the mixing of ejecta with the circumstellar and interstellar medium, so the emerging class of metal-rich MMSNRs is still not properly understood. This is why comparisons between models and observation have focused mostly on morphological issues, with the general comment that the presence of the additional ejecta component may reconcile the discrepancy between model and observed profile (as in the case of W44, Shelton et al. 2004).

The mixed morphology category of SNRs (and especially the emerging subclass of metal-rich MMSNRs) can be understood more clearly if we have a large sample of objects with well 
studied characteristics. In particular, the accurate determination of density, temperature, and abundances profile for MMSNRs would allow us to perform an accurate comparison both with traditional models and with a new magnetohydrodynamical (MHD) model of shocked ISM and ejecta inside MMSNRs, which is the subject of a forthcoming publication.

We systematically reviewed the MMSNRs listed in Lazendic $\&$ Slane (2006) with the primary goal of measuring the abundances in the inner region of the remnants and other X-ray properties. In this paper, we review the XMM-Newton archive observations of two bright MMSNRs, IC 443 and G166.0+4.3, and attempt to derive the distribution of temperature and metal abundances inside these remnants. Both our targets are mentioned in the list of Lazendic \& Slane (2006) as MMSNRs with standard metal abundances. However, Troja et al. (2006) and Troja et al. (2008) studied in detail the X-ray emission of IC 443, and found enhanced metal abundances in a remarkable ring feature around the pulsar wind nebula, in the southern part of the remnant, which they attributed to emission from shocked ejecta. The compilation of MMSNRs with enhanced metal abundances needs therefore to be updated with new results from deep X-ray observations of MMSNRs. In the light of these new observational results, traditional models should be verified and revised as necessary.

The paper is organized as follows. In Sects. 2 and 3, we present the results obtained form XMM-Newton archive observations of IC 443 and G166.0+4.3, respectively. In Sect. 4, we present the comparison of our results with the standard models for MMSNRs, namely the evaporating clouds White \& Long (1991) model and the radiative SNR model of Cox et al. (1999). In Sect. 5, we present the conclusion of our work.

\section{IC 443}

IC 443 has been extensively studied and observed in several bands and, for this reason, is a case study for the interaction between SNR shocks and molecular clouds. Recent X-ray studies include those of Troja et al. (2008), Bykov et al. (2008), and Troja et al. (2006), while infrared and radio studies include Noriega-Crespo et al. (2008), Bykov et al. (2008), Lee et al. (2008), Rosado et al. (2007), Leahy (2004), and references therein. The remnant was classified as mixed-morphology by Rho \& Petre (1998), based on observations with the ROSAT satellite. Troja et al. (2006) and Troja et al. (2008) confirmed the centrally peaked morphology between 0.5 and $5 \mathrm{keV}$, and a bright X-ray peak close to $06^{\mathrm{h}} 17^{\mathrm{m}} 09^{\mathrm{s}}+22^{\circ} 45^{\mathrm{m}}$ in the $1.4-5 \mathrm{keV}$ (Fig. 1). However, they note that, at very soft energies (0.3$0.5 \mathrm{keV})$, the remnant exhibites an incomplete shell extending from $\mathrm{N}$ to $\mathrm{E}$, corresponding to one of the regions of interaction with a large cloud. They also investigated the spatial distribution of the metals inside this remnant, using the technique of the equivalent width images and demonstrated that the distribution of $\mathrm{Si}$ and $\mathrm{S}$ is far from being homogeneous. In particular, a ring of metal-rich plasma surrounding the pulsar wind nebula is evident in the equivalent width images of these two elements (see their Fig. 3). The northern region of the remnant, coincident with a maximum in the hard X-ray image (Fig. 1), is also characterized by a large value of the Si and S equivalent width, suggesting that it has a higher metal abundances with respect to surrounding regions. In the following, we focus on this region.

The data that we use in this work are part Calibration and Performance Verification phase of the XMM-Newton satellite (Jansen et al. 2001), for a total of 6 observations of IC 443. We have also used the public archive observation 0301960101. The

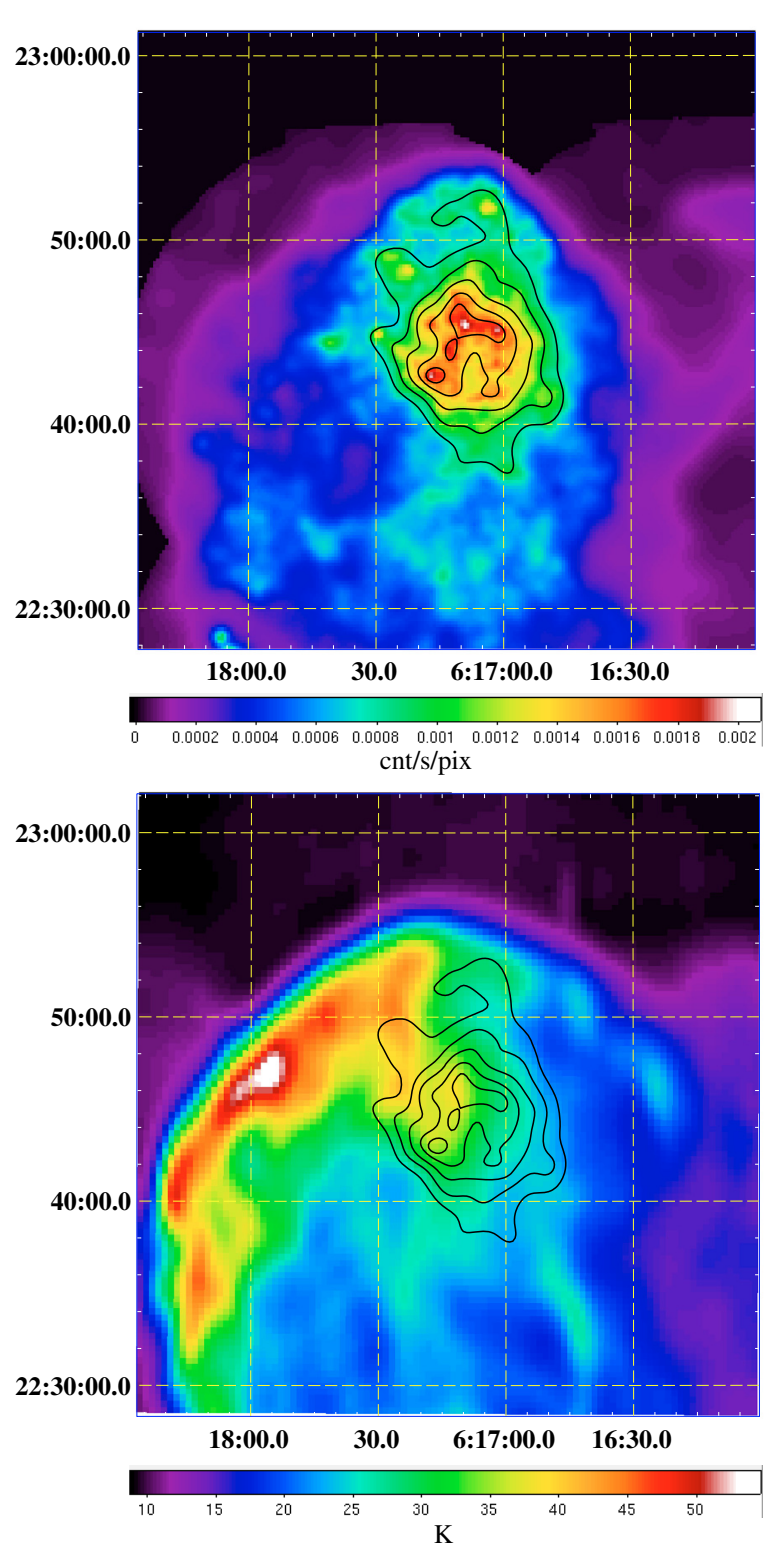

Fig. 1. Top: XMM-Newton EPC mosaic of the northern region of the IC 443 supernova remnant in the $1.4-5.0 \mathrm{keV}$ band. The image is background subtracted and vignetting corrected. The pixel size is 10 arcsec. Five contours levels at $8,10,12,14$, and $16 \times 10^{-4} \mathrm{cnt} \mathrm{s}^{-1} \mathrm{pix}^{-1}$ are overlaid. These contours have been used as boundaries for the 5 spectral extraction regions discussed in the text. Bottom: same region at $1420 \mathrm{MHz}$ (total intensity map) with X-ray contours overlaid (adapted from Leahy 2004).

datasets that we used are the same datasets used by Troja et al. (2008). We screened both PN and MOS data using the algorithm suggested by Snowden \& Kuntz (2007).

To study the profile of the thermodynamical and chemical parameters of this remnant, we chose 5 spectral regions defined in terms of contours of iso-surface brightness, centered on the bright northern region, as shown in Fig. 1. For each region, we computed the mean distance to the X-ray peak by finding the average distance for all pixels in that region. XMM-Newton PN and MOS spectra were extracted using the software SAS 7.1 and corrected for detector non-uniformity using the task evigweight. Troja et al. (2006) demonstrated that the X-ray emission in the IC 443 interior consists of two thermal components, a soft one of temperature in the range $0.3-0.7 \mathrm{keV}$, in a non-equilibrium of 


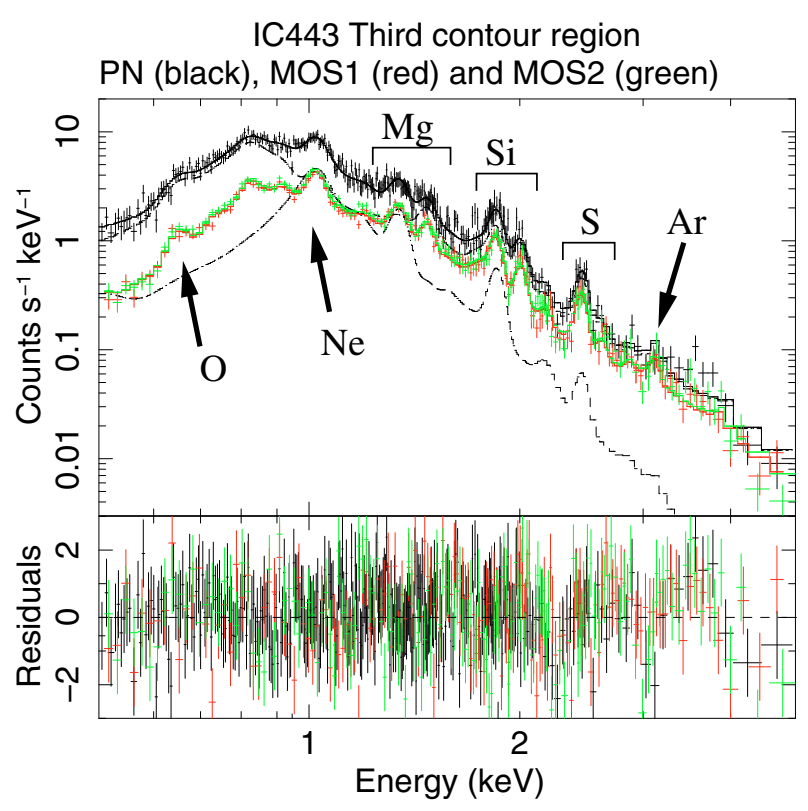

Fig. 2. XMM-Newton EPIC spectrum of the third contour region in Fig. 1 (average distance 2.8' in Fig. 3). The best fit two thermal component model is shown as continuous line (with residual in lower panel), while individual components are shown in dashed black lines for the PN spectrum only. Emission lines from $\mathrm{O}(0.6-0.8 \mathrm{keV}), \mathrm{Ne}(\sim 1 \mathrm{keV}), \mathrm{Mg}$ $(\sim 1.4 \mathrm{keV}), \mathrm{Si}(1.8-2 \mathrm{keV}), \mathrm{S}(\sim 2.5 \mathrm{keV})$ and $\mathrm{Ar}(\sim 3 \mathrm{keV})$ are visible.

Ionization (NEI) state, and a second with $k T=1.1-1.8 \mathrm{keV}$, in equilibrium of ionization (CIE) state. The soft component has been associated with the shocked interstellar material, and the hot component with the shocked stellar ejecta. Therefore, we used the same emission models, namely the MEKAL model (Mewe et al. 1985) and the VNEI (Borkowski et al. 2001) model of XSPEC v11.3.2 (Arnaud 1996). We fixed the chemical abundances of the soft component to solar values, and allowed the abundances of the hot component to vary. The adopted models represent an approximation to the true conditions inside this complex remnant. However, this approach allows us to measure quantitatively the emission-measure-weighted temperature and metal abundances of each component in a spectrum. By using the region layout shown in Fig. 1, we may also derive meaningful constraints on the measured quantities related to the radial distance of the regions.

In Fig. 2, we show an example of a spectrum, while the complete results of the spectral analysis are reported in Fig. 3 for the 5 spectral regions, displayed in order of increasing distance from the putative center. The temperature does not show large variations, indicating that thermal conduction must be efficient. The innermost regions are characterized by a higher metal abundances for $\mathrm{Ne}, \mathrm{Mg}$ and $\mathrm{Si}$. In these cases, the profiles show a decreasing trend with increasing distance from the center. While $\mathrm{Ne}$ and $\mathrm{Mg}$ retain super-solar abundances in most regions, $\mathrm{Si}$ is above solar abundance only in the two innermost regions. On the other hand, $\mathrm{S}$ and Fe appear to have more uniform abundances, about 1.0 and 0.3 respectively. The thermodynamical parameters of both the components agree in general with the findings of Troja et al. (2006). The abundances patterns are generally consistent with the ones found by Troja et al. (2008) in the south region of the remnant, but smaller in value than those of the remarkable ejecta ring surrounding the pulsar wind nebula found by them. Troja et al. (2008) also compared the $\mathrm{Mg} / \mathrm{Si}$, $\mathrm{S} / \mathrm{Si}$, and $\mathrm{Fe} / \mathrm{Si}$ ratios of the ring with the predictions of both the

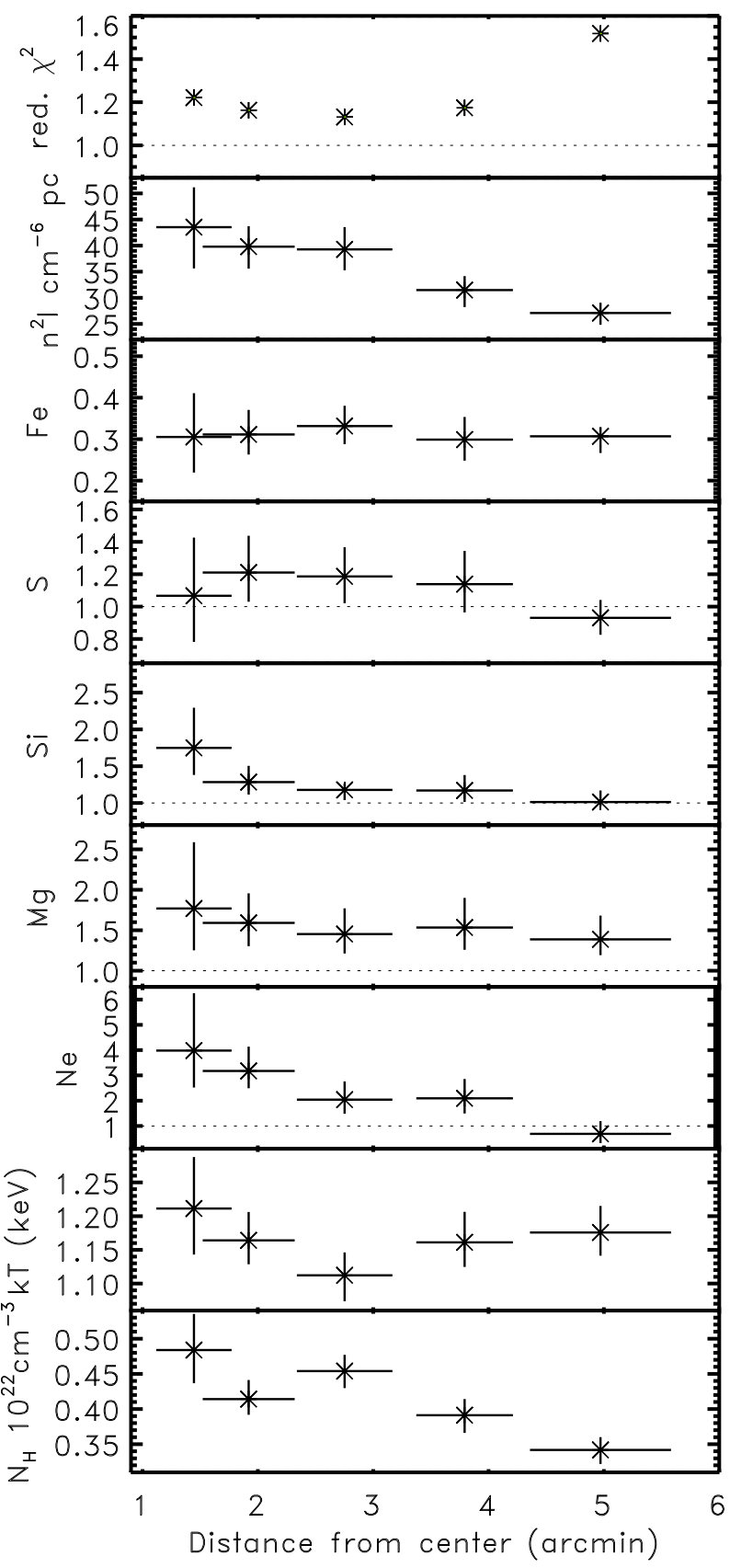

Fig. 3. Results of the spectral fittings to the hard thermal component obtained in the 5 iso-brightness regions in IC 443. See Fig. 1 for the position of the contours levels used in the region definition. Abundances are relative to solar values. The abscissa contains the average distance from the center (horizontal error bars are standard deviations). Vertical error bars are $2 \sigma$ uncertainties. The normalization of the spectrum is expressed in terms of $n^{2} l$, where $n$ is the plasma density and $l$ is its extension along the line of sight.

nucleosynthesis model of core-collapse (Woosley \& Weaver 1995) and type Ia (Badenes et al. 2003) supernovae, finding closer agreement with the former model. We verified that $\mathrm{Mg} / \mathrm{Si}$, $\mathrm{S} / \mathrm{Si}$ and $\mathrm{Fe} / \mathrm{Si}$ ratios found by us in the innermost spectral region in Fig. 1 agree with those in the ring region, and thus we concluded that our results also support the idea that IC 443 had a type II progenitor. 


\section{G166.0+4.3 (VRO 42.05.01)}

G166.0+4.3 is a supernova remnant in the anti center direction, whose X-ray and radio morphology inspired considerable interest. Burrows \& Guo (1994) and Guo \& Burrows (1997) analyzed the ROSAT and ASCA X-ray observations of this remnant, finding a centrally peaked morphology that appeared to be completely enclosed within the radio emission of this object. The radio emission, in turn, has a limb-brightened double-shell morphology, which has a small diameter half-shell at NE (about 30', a.k.a. the shell) and a larger incomplete shell at SW (a.k.a. the wing, Pineault et al. 1985; Pineault et al. 1987; Leahy \& Tian 2005, and references therein).

G166.0+4.3 was observed by XMM-Newton on 21 February 2003 (ID 0145500101) and on 23 February 2003 (ID 0145500201). The first observation was pointed at the western part of the remnant (the "wing"), and had a total PN exposure time, after the screening procedure, of $5.0 \mathrm{ks}$, while the second targeted the small incomplete shell at the east. This latter pointing was severely contaminated by high energy proton flares and the net PN exposure time is only $1 \mathrm{ks}$, and was not used in the spectral analysis.

A mosaic map of this remnant in the band $0.3-5.0 \mathrm{keV}$, obtained using the two XMM-Newton archive observations, is shown in Fig. 4, which also includes the $1420 \mathrm{MHz}$ radio map of the Canadian Galactic Plane Survey (Taylor et al. 2003). The $\mathrm{X}$-ray morphology derived by previous observations was confirmed, and the bright region (named "western bright knot" by Burrows \& Guo 1994) was resolved for the first time, showing an elongation in the NW-SE direction. In the light of its radio and X-ray morphology, this remnant may be considered part of the sub-class of mixed-morphology (MM) SNRs introduced by Rho \& Petre (1998). The explanation of the peculiar radio morphology dates to Pineault et al. (1987) and involves an explosion in a moderately dense medium, followed by the breaking of the shock in a rarefied hot tunnel, and by the interaction with a denser medium again. Guo \& Burrows (1997) made the so far only measurement of metal abundances in the X-ray spectrum of G166.0+4.3, finding a substantial underabundance of $\mathrm{Mg}, \mathrm{Si}$, and $\mathrm{Fe}$ in the western bright knot and in the SE and NE of the wing.

To study the abundances in the centrally peaked region of G166.0+4.3, we tried to characterize the thermal properties in the plasma of this remnant by selecting 4 interesting regions for spectral analysis, which are shown in Fig. 4. Regions 1, 2, and 3 are similar to regions 1, 2 and 3 of Guo \& Burrows (1997), but are considerably smaller and point towards bright features, and are therefore much less affected by background (also considering the smaller XMM-Newton PSF compared to the ASCA one). In each region, we fitted the X-ray spectra with a Mewe et al. (1985) spectral model modified by interstellar absorption, allowing the abundances of $\mathrm{O}, \mathrm{Ne}, \mathrm{Mg}, \mathrm{Si}, \mathrm{S}$, and $\mathrm{Fe}$ to vary. In Fig. 5, we show, as an example, the spectrum of region 1. The results of the spectral analysis, presented in Fig. 6, indicate small variation in temperature (of up to $10 \%$ ) and large variation in interstellar absorption (of up to $50 \%$ ). The variation patterns appear to agree more closely with those found by Burrows \& Guo (1994) than those of Guo \& Burrows (1997). The regions appear to be characterized by an underabundance of $\mathrm{O}$ and $\mathrm{Si}$ and an overabundance of $\mathrm{S}$, while $\mathrm{Ne}, \mathrm{Mg}$, and $\mathrm{Fe}$ appear to have about solar abundance values. Our $\mathrm{Mg}, \mathrm{Si}$, and $\mathrm{Fe}$ abundance measurements agree with those of Guo \& Burrows (1997), who also measured subsolar abundances of these elements. A NEI thermal model does not improve the fits for these regions,
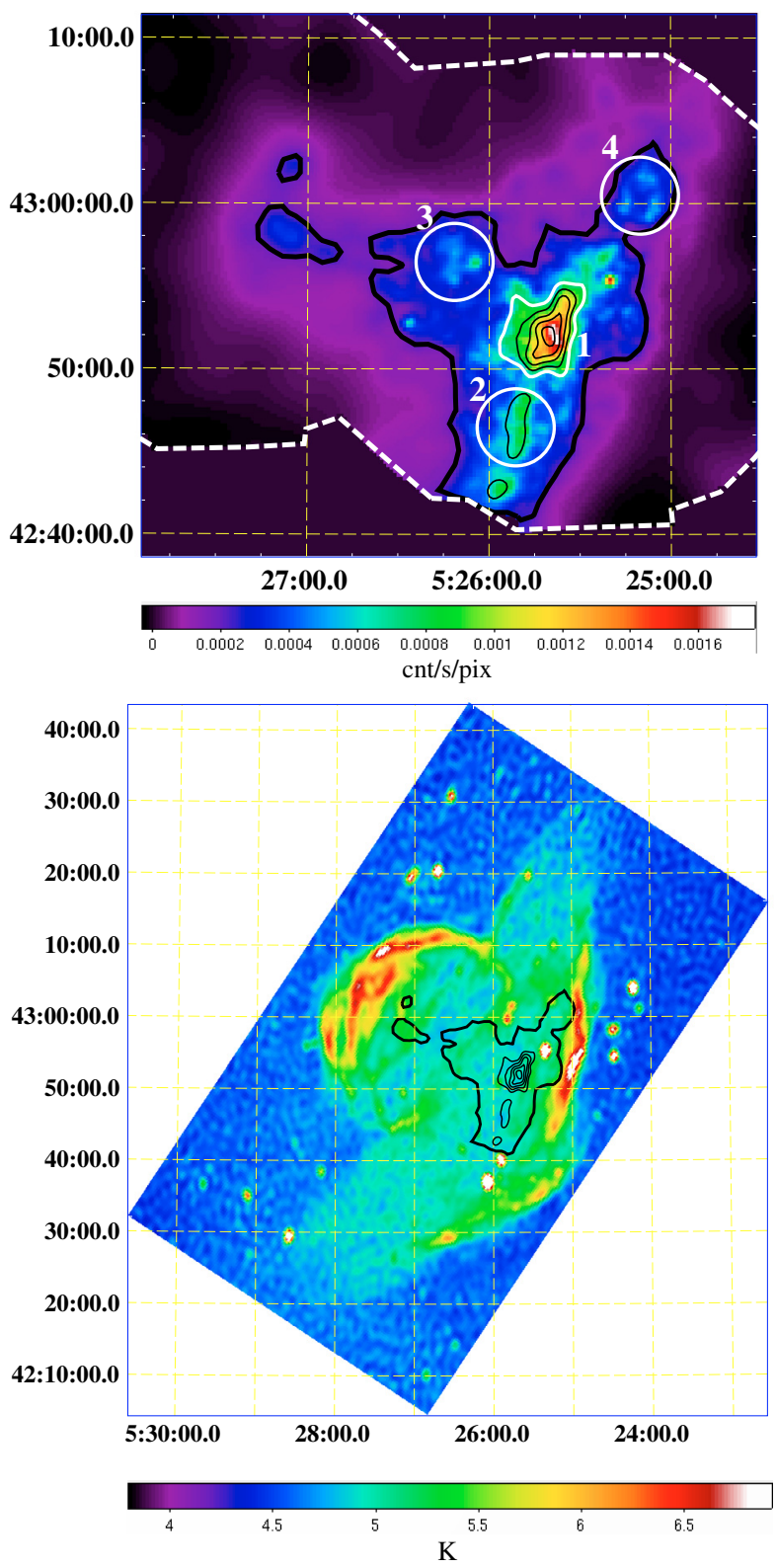

Fig. 4. Top: XMM-Newton EPIC images of G166.0+4.3 in the 0.3$5 \mathrm{keV}$ background subtracted and vignetting corrected. Black thick contour is at $0.2 \times 10^{-3} \mathrm{cnt} \mathrm{s}^{-1} \mathrm{pix}^{-1}$ and black thin contours correspond to $0.6,0.8,1.0,1.2$ and $1.5 \times 10^{-3} \mathrm{cnt} \mathrm{s}^{-1} \mathrm{pix}^{-1}\left(1\right.$ pixel $\left.=12^{\prime \prime} \times 12^{\prime \prime}\right)$. Numbers mark the spectral extraction region used in the text (Region 1 is defined by the outermost thin surface brightness contour). The XMMNewton EPIC field of view is marked in white (two observations combined). Bottom: $1420 \mathrm{MHz}$ CGPS DRAO radio image of G166.0+4.3 (adapted from Leahy \& Tian 2005). X-ray contours of the top panel are shown in black.

giving a ionization time $\tau \gtrsim 10^{12} \mathrm{~s} \mathrm{~cm}^{-3}$ and abundances similar to those obtained with the equilibrium model.

We investigated whether a radial gradient in metal abundances exists inside the bright region 1 of the remnant (the "western bright knot"), in a similar way of the analysis in Sect. 2 for IC 443, but, due to the low quality of the data in this case, the results do not provide tight constraints on metal abundances. 


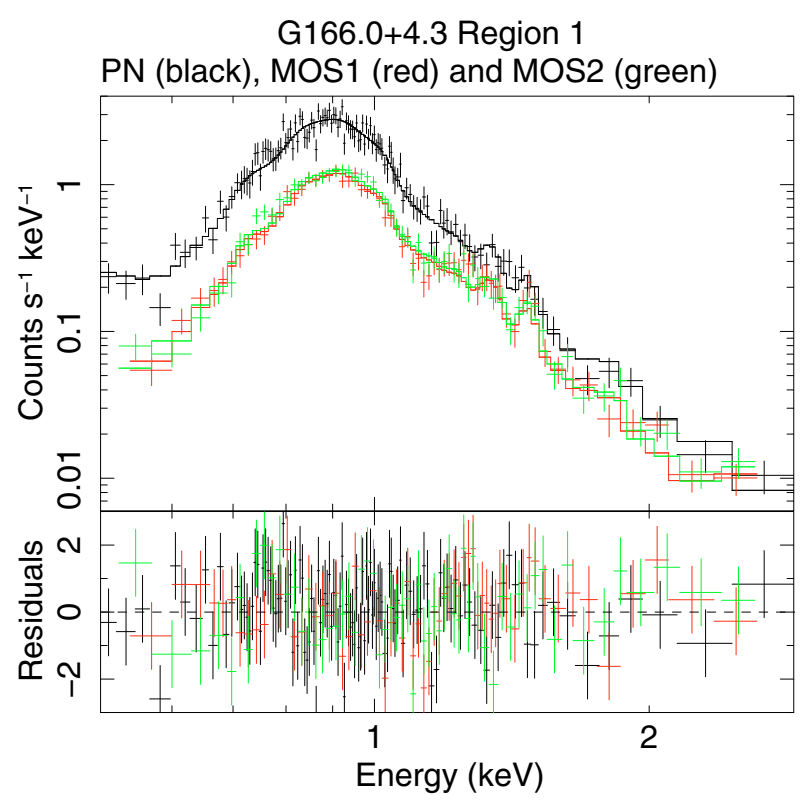

Fig. 5. XMM-Newton EPIC spectrum of the G166.0+4.3 Region 1 in Fig. 4. The best fit thermal model is shown as continuous line (with residual in lower panel).

\section{Discussion}

In Lazendic \& Slane (2006) IC 443 and G166.0+4.3 were listed as standard abundances MMSNRs. However, the spatially resolved spectral analysis we did on the bright X-ray central regions of IC 443 confirmed that this object belongs to the subclass of metal-rich mixed-morphology remnants. In the case of G166.0+4.3, no compelling evidence of enhanced metal abundances exists apart from sulfur in the outer regions. The short exposure time of the XMM-Newton observation of the G166.0+4.3 prevented us from studying possible trends in the metal abundances of the remnant core. Our results suggest that we should review the other MMSNRs in the list of Lazendic \& Slane (2006) to better measure chemical abundances of all known remnant of similar type. Other MMSNRs may be erroneously cataloged as solar-type abundances objects.

It is not easy to determine whether the high metal abundances measured in some MMSNRs are typical of this class, or if they correspond to an evolutionary phase in the life of these objects, or depend on the initial remnant composition. From the theoretical point of view, both the evaporating-cloud model of White \& Long (1991) and the radiative SNR model Cox et al. (1999), used traditionally to explain the centrally peaked thermal X-ray morphologies, are of limited help in understanding the subclass of metal-rich MMSNRs, since they do not consider in detail the mixing between ejecta and shocked material inside the remnants. However, these models have often been used to interpret the X-ray emission of some MMSNRs (e.g., Bocchino \& Bandiera 2003; Lazendic \& Slane 2006), and should therefore be compared with our own results in understanding their limitations.

We first consider the evaporating-cloud model of White \& Long (1991), in which the X-ray central enhancement is due to material ablated by shock-heated clouds inside the remnant. The models depend on the traditional parameters $E$ (the explosion energy), $\rho$ (the preshock ISM density), and $t$ (the age of the remnant), and two parameters describing the evaporating clouds, namely $C$ (the ratio of mass in clouds to mass in

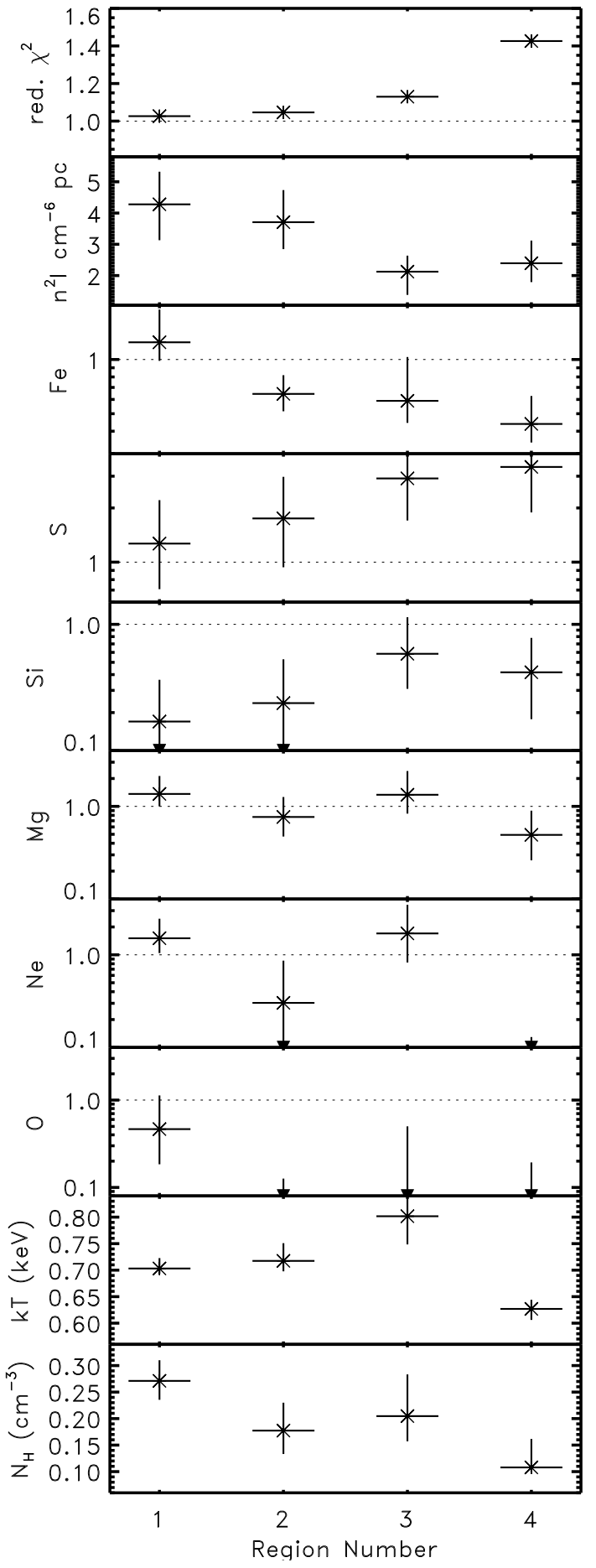

Fig. 6. Spectral fitting results of regions $1-4$ in G166.0+4.3. See Fig. 4 for the position of the regions. Black arrows indicates a lower limit of 0 .

the intercloud medium) and $\tau$ (the ratio of the cloud evaporation time-scale to the SNR age). With an appropriate choice of normalized quantities for the radial profiles (for our study, we consider the density normalized to the post-shock density, $\rho / \rho_{s}$, the temperature normalized to the shell temperature $T / T_{s}$, and the normalized emission measure $E M\left(\pi R_{s}^{2}\right) / E M($ tot $)$; see their Fig. 4), they show that the dependence on $E, \rho_{0}$, and $t$ can be masked out in the presentation of the results. To ease the comparison with observations, the authors discussed the asymptotic behavior of their model when $C$ and $\tau \rightarrow \infty$, in which case the model only depends on the ratio $C / \tau$. In Fig. 7, we show the 


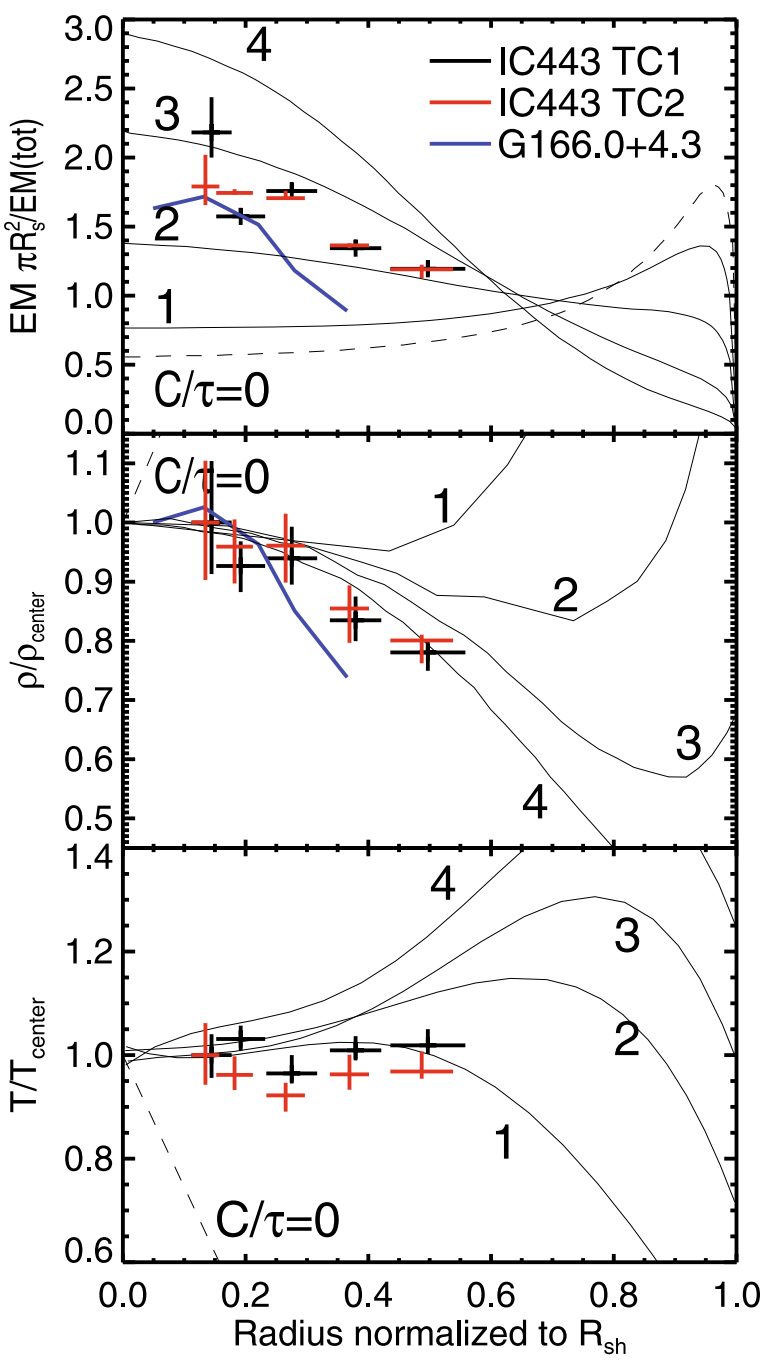

Fig. 7. Comparison between the White \& Long (1991) evaporatingcloud model and the observed profiles in IC 443 and G166.0+4.3. Top: normalized emission measure (see text for explanation). Middle: density normalized to central value. Bottom: post-shock temperature normalized to central value. Black and red crosses are IC 443 low and high temperature component data with uncertainties, respectively; blue curves are G166.0+4.3 data. Black curves are White \& Long (1991) profiles for models with $C / \tau=0$ (dashed), $1,2,3$ and 4 . The distance from the center is normalized to the shell radius (assumed 10' for IC 443 and $\sim 6.5^{\prime}$ for $\mathrm{G} 166.0+4.3$, see text for details).

normalized temperature, density and emission measure for the models with $C / \tau=0,1,2,3$ and 4 . To ease the comparison with the data further, we decided to normalize the temperature and density to their respective central values, instead of their shell values, as in the work of White \& Long (1991). In our observations (and in general in all MMSNRs observations), the low $\mathrm{X}$-ray surface brightness of the shell prevents an accurate spectral analysis from being possible, so it is particularly difficult to estimate the shell values. On the other hand, the central regions are by definition the brightest, and accurate results may be achieved here. An additional concern is the shell radius used to normalize both the abscissa of the profiles and the emission measure. Both IC 443 and G166.0+4.3 have morphologies that are far from being circular symmetric and offset X-ray peaks, so for each of these objects, it is particularly difficult to estimate a single shell radius. For IC 443, we adopted a radius of $10^{\prime}(\sim 4.3 \mathrm{pc}$ at $1.5 \mathrm{kpc}$ distance), which is the average distance of the radio shell from the X-ray peak in the East, North, and West of the remnant. For G166.0+4.3, we used $\sim 6.5^{\prime}(\sim 13$ pc at $4.5 \mathrm{kpc}$ distance), which is the distance to the east radio wing.

In Fig. 7, we also plot the derived values for the low and high temperature component of IC 443 and the single thermal component of G166.0+4.3. The profiles of G166.0+4.3 appear to be steeper than the profiles of IC 443. In general, there is a good agreement between the normalized emission-measure and that of the model with $C / \tau=2-3$ for both objects, even if the uncertainties in the adopted shell radius can affect the final results. However, we emphasize that the density and temperature profiles are not entirely consistent with the emission measure profiles. In case of IC 443, the density profile would be in closer agreement with $C / \tau \geq 4$, while the temperature profile would be with $C / \tau<1$. An inconsistency is present in both the thermal components of IC 443, which also holds for the density of G166.0+4.3 implying that $C / \tau>4$. Conversely, we could use the density profile to find a good fit to the model by varying the shell radius within a $\pm 50 \%$ range, but the emission measure and temperature profile would remain inconsistent. We verified for instance that the emission measure would be far more centrally peaked than observed. Finally, we note that the central peak in both the observed density profiles seems to exclude evaporatingcloud models with low values of $\tau$ (cf. Fig. 1 in White \& Long 1991).

In summary, the comparison with the evaporating-cloud model infers high values of $\tau$, but does not constrain tightly the true actual value of $C$ and $\tau$; it also indicates that the observed density profiles are usually steeper than predicted by the model reproducing the emission measure profiles more successfully, a situation that cannot be explained in terms of an ejecta component on top to the evaporating-cloud component. If it was so, in fact, we would expect the density profile to be shallower than the model, because of the enhancement in density provided by the ejecta. We therefore conclude that the X-ray morphology of these remnants is inconsistent with the evaporating-cloud model. The temperature profiles, although not entirely consistent with the model, appear to indicate very limited variations, and therefore points toward efficient thermal conduction inside both of the remnants. The failure of the cloud evaporation model and the high efficiency of thermal conduction suggest an explanation in terms of the entropy-mixed thermally conductive model developed by Shelton et al. (2004) to explain the observational data of MMSNR W44, possibly amended to take into account the peculiar environment in which these remnants expand. A detailed numerical model of the metal-rich MMSNRs that takes into account mixing between the ejecta and shocked ISM material, thermal conduction, and a realistic model of the environment, is required to verify this scenario.

The comparison with the radiative model of Cox et al. (1999) can be completed more straightforwardly following Lazendic \& Slane (2006) by assuming that the current shock radius is the radius at which the remnant enters the radiative stage. Following the relations of Cox et al. (1999), this yields a lower limit to the ambient and central densities. Using realistic radius values, as used in our comparison with the cloudy ISM model, we derive ambient density lower limits of the order of $10 \mathrm{~cm}^{-3}$. While it is true that, in both remnants, there is substantial evidence of expansion in dense environments, it is also unlikely that the expansion always occurred inside a medium so dense. For IC 443, Troja et al. (2006) argued that the remnant evolved inside wind-blown bubble, hypothesis originally suggested by Braun \& Strom (1986), while for G166.0+4.3, Gaensler (1998) argued that the remnant had expanded into a low-density hot 
tunnel before encountering recently a high density region. The hot tunnel location was inferred to coincide with that of the Xray emission peak. In both cases, it is unlikely that a radiative model assuming a long evolution inside a dense medium is applicable. This is also the case whenever there is evidence of a massive progenitor star, which usually have strong pre-supernova winds. Chen et al. (2008) proposed that the X-ray emission of Kes 27 MMSNR could be explained in terms of a shock reflected by a cavity wall, a mechanism that may operate also in IC 443 and G166.0+4.3, given the observational evidences about their environments.

\section{Summary and conclusion}

We analyzed on XMM-Newton X-ray observations of the two supernova remnants IC 443 and G166.0+4.3, focussing on the nature of their centrally peaked thermal X-ray emission above $1 \mathrm{keV}$. We confirmed that the X-ray morphology of these remnants implies that they belong to the class of mixed morphology SNRs. In contrast to previous results, we measured the chemical abundances of the bright central peak of IC 443 to be above solar, which infers that this object is a member of the subclass of metal-rich MMSNRs. For G166.0+4.3, there is no evidence of enhanced metal abundances, except maybe for sulfur in the outer regions, but the limited quality of the data prevented us from performing spatially resolved spectroscopy of the central peak.

We derived profiles of metallicity, temperature, density, and emission measure, and compared them with the predictions of the White \& Long (1991) and Cox et al. (1999) models, traditionally used to explain the MMSNR morphology in other objects. We found that the observed profiles are inconsistent with the models, and we argued that more detailed modeling including the mixing of ISM and ejecta material is required to explain the observations more accurately. The entropy-mixing model of Shelton et al. (2004) appears to be the most promising starting point for more detailed modeling, although the effects of the complex environment in which the MMSNRs seem to be located (Lazendic \& Slane 2006) and the projection effects discussed by Petruk (2001) should be taken into account. Temperatures, density, and chemical abundances profiles of MMSNRs are therefore the key observational constraints to be compared with future models.

Acknowledgements. We thank D. Leahy for providing us with the $1420 \mathrm{MHz}$ total intensity map of IC 443 in electronic format. We also thank O. Petruk and
S. Orlando for useful comments on this work. This work makes use of results produced by the PI2S2 Project managed by the Consorzio COMETA, a project co-funded by the Italian Ministry of University and Research (MIUR) within the Piano Operativo Nazionale "Ricerca Scientifica, Sviluppo Tecnologico, Alta Formazione" (PON 2000-2006). More information is available at http: //wwW . consorzio-cometa.it.

\section{References}

Arnaud, K. A. 1996, in Astronomical Data Analysis Software and Systems V, ed. G. H. Jacoby, \& J. Barnes, ASP Conf. Ser., 101, 17

Badenes, C., Bravo, E., Borkowski, K. J., \& Domínguez, I. 2003, ApJ, 593, 358 Bocchino, F., \& Bandiera, R. 2003, A\&A, 398, 195

Borkowski, K. J., Lyerly, W. J., \& Reynolds, S. P. 2001, ApJ, 548, 820

Braun, R., \& Strom, R. G. 1986, A\&A, 164, 193

Burrows, D. N., \& Guo, Z. 1994, ApJ, 421, L19

Bykov, A. M., Krassilchtchikov, A. M., Uvarov, Y. A., et al. 2008, ApJ, 676, 1050

Chen, Y., Seward, F. D., Sun, M., \& Li, J.-T. 2008, ApJ, 676, 1040

Chevalier, R. A. 1999, ApJ, 511, 798

Cox, D. P., Shelton, R. L., Maciejewski, W., et al. 1999, ApJ, 524, 179

Gaensler, B. M. 1998, ApJ, 493, 781

Guo, Z., \& Burrows, D. N. 1997, ApJ, 480, L51

Hwang, U., Petre, R., \& Flanagan, K. A. 2008, ApJ, 676, 378

Jansen, F., Lumb, D., Altieri, B., et al. 2001, A\&A, 365, L1

Katsuda, S., Tsunemi, H., Miyata, E., et al. 2008, PASJ, 60, 107

Lazendic, J. S., \& Slane, P. O. 2006, ApJ, 647, 350

Leahy, D., \& Tian, W. 2005, A\&A, 440, 929

Leahy, D. A. 2004, AJ, 128, 1478

Lee, J.-J., Koo, B.-C., Yun, M. S., et al. 2008, AJ, 135, 796

Mewe, R., Gronenschild, E. H. B. M., \& van den Oord, G. H. J. 1985, A\&AS, 62,197

Miceli, M., Bocchino, F., \& Reale, F. 2008, ApJ, 676, 1064

Noriega-Crespo, A., Hines, D. C., Gordon, K., et al. 2008, ArXiv e-prints, 804

Park, S., Hughes, J. P., Burrows, D. N., et al. 2003a, ApJ, 598, L95

Park, S., Hughes, J. P., Slane, P. O., et al. 2003b, ApJ, 592, L41

Petruk, O. 2001, A\&A, 371, 267

Pineault, S., Pritchet, C. J., Landecker, T. L., Routledge, D., \& Vaneldik, J. F. 1985, A\&A, 151, 52

Pineault, S., Landecker, T. L., \& Routledge, D. 1987, ApJ, 315, 580

Rho, J., \& Petre, R. 1998, ApJ, 503, L167

Rho, J., \& Borkowski, K. J. 2002, ApJ, 575, 201

Rosado, M., Arias, L., \& Ambrocio-Cruz, P. 2007, AJ, 133, 89

Shelton, R. L., Cox, D. P., Maciejewski, W., et al. 1999, ApJ, 524, 192

Shelton, R. L., Kuntz, K. D., \& Petre, R. 2004, ApJ, 611, 906

Slane, P., Smith, R. K., Hughes, J. P., \& Petre, R. 2002, ApJ, 564, 284

Snowden, S., \& Kuntz, K. 2007, XMM-Newton ESAS manual,

ftp: //xmm. esac.esa.int/pub/xmm-esas/xmm-esas.pdf, 2

Taylor, A. R., Gibson, S. J., Peracaula, M., et al. 2003, AJ, 125, 3145

Tilley, D. A., Balsara, D. S., \& Howk, J. C. 2006, MNRAS, 371, 1106

Troja, E., Bocchino, F., \& Reale, F. 2006, ApJ, 649, 258

Troja, E., Bocchino, F., Miceli, M., \& Reale, F. 2008, A\&A, 485, 777

White, R. L., \& Long, K. S. 1991, ApJ, 373, 543

Woosley, S. E., \& Weaver, T. A. 1995, ApJS, 101, 181 